\title{
Inhibition of pre-B cell colony-enhancing factor attenuates inflammation induced by hyperoxia in EA.hy926 cells
}

\author{
JIA YU HE, HUAN JIN CUI, LI JUN TANG, JINWEN CHEN and WEI MIN HUANG
}

Department of Neonatology, Nanfang Hospital, Southern Medical University, Guangzhou, Guangdong 510515, P.R. China

Received November 6, 2015; Accepted June 9, 2017

DOI: $10.3892 /$ ijmm.2017.3045

\begin{abstract}
The aim of this study was to investigate the role of pre-B cell colony-enhancing factor $(\mathrm{PBEF})$ in the pathogenesis of bronchopulmonary dysplasia (BPD) using an established cell model of BPD. For this purpose, EA.hy926 cell cultures were divided into 4 groups as follows: the air group as the blank control, the hyperoxia group, the hyperoxia plus PBEF siRNA group and the hyperoxia plus scramble siRNA group. Cell viability and the generation of reactive oxygen species (ROS) were determined using respective kits. Moreover, the protein and mRNA expression levels of PBEF, interleukin-8 (IL-8) and tumor necrosis factor- $\alpha(\mathrm{TNF}-\alpha)$ were also detected by corresponding methods. Compared with the hyperoxia group, the ROS levels in the hyperoxia plus PBEF siRNA group were significantly reduced $(\mathrm{P}<0.01)$. The silencing of $\mathrm{PBEF}$ increased cell viability compared with the hyperoxia group. The protein and mRNA expression levels of PBEF, IL-8 and TNF- $\alpha$ were all decreased in the hyperoxia plus PBEF siRNA group compared with the hyperoxia group $(\mathrm{P}<0.01)$. Our study thus demonstrates that the inhibition of PBEF attenuates oxidative stress and inflammation induced by hyperoxia in EA.hy926 cells, suggesting that PBEF may be a potential diagnostic and therapeutic target, which may be used for the development of novel treatment strategies for BPD.
\end{abstract}

\section{Introduction}

Bronchopulmonary dysplasia (BPD) is a chronic lung disease, with a high morbidity and mortality in pre-term infants. It is the cause of prolonged hospitalization with serious social and economic consequences. Although the pathogenic and genetic basis of BPD remains incompletely understood,

Correspondence to: Professor Wei Min Huang, Department of Neonatology, Nanfang Hospital, Southern Medical University, 1838 Guangzhou Avenue North, Guangzhou, Guangdong 510515, P.R. China

E-mail: drhwm2003@hotmail.com

Key words: bronchopulmonary dysplasia, pre-B cell colonyenhancing factor, hyperoxia, oxidative stress, inflammatory response, interleukin-8, tumor necrosis factor- $\alpha$ studies have suggested that inflammation-mediated damage and oxidative stress play an important role in the development of BPD (1-4). A number of cytokines, such as tumor necrosis factor- $\alpha$ (TNF- $\alpha$ ), interleukin (IL)-8, IL-1 $\beta$, IL-6, intercellular adhesion molecule-1 (ICAM-1) and nuclear factor- $\kappa \mathrm{B}(\mathrm{NF}-\kappa \mathrm{B})$ have been proposed to be involved in the developmen tof lung inflammation, and to be closely related to the pathogenesis of BPD (5).

Pre-B-cell colony-enhancing factor (PBEF) was first identified by Samal et al in 1994 as a putative cytokine (6), which was involved in the maturation of B-cell precursors in the presence of IL-7, and also known as NAMPT. PBEF is synthesized and secreted by many types of cells, such as activated lymphocytes, epithelial cells and neutrophils (7). It was previously demonstrated that PBEF (visfatin) exerts pro-inflammatory effects by upregulating the production of the pro-inflammatory cytokines, IL-1 $\beta$, TNF- $\alpha$, IL-6, in human monocytes (8). Ye et al reported the first findings of PBEF expressed in lung tissues and to be overexpressed in acute lung injury (ALI) (9). Furthermore, PBEF is considered to be associated with the regulation of inflammation and endothelial barrier dysfunction in ALI (10). Kim et al demonstrated that PBEF stimulated reactive oxygen species (ROS) generation in endothelial cells, and antioxidants blocked PBEF-induced NF- $\kappa \mathrm{B}$ activation and cell adhesion molecule (CAM) expression (11). PBEF expression is upregulated and plays an important role during the development of a variety of inflammation-related diseases, including ALI, sepsis, rheumatoid arthritis and atherosclerosis (7-9,12,13). Based on these results, we hypothesized that PBEF may be a potential modulator in the pathogenesis of BPD.

The EA.hy926 cell line is an permanent human endothelial cell line, which was derived from the fusion of human umbilical vein endothelial cell (HUVEC) line and A549 cell line (14). The EA.hy926 cell line has highly differentiated functions that are characteristic of lung adenocarcinoma cells and the vascular endothelium (15). Xu et al used these alveolar endothelial cells to construct the alveoli in vitro model (16). The EA.hy926 cells have been widely applied in the study of inflammation, oxidative stress and protein expression (17-20).

In the present study, we aimed to explore the role of PBEF in BPD and its mechanisms of action by establishing an in vitro cell model of BPD. siRNA targeting PBEF (PBEF siRNA) was used to knockdown PBEF in the EA.hy926 cells in order to observe the effects of PBEF on inflammation and apoptosis caused by hyperoxia. 


\section{Material and methods}

Reagents and materials. The triple gas mixture $\left(60 \% \mathrm{O}_{2}\right.$, $5 \% \mathrm{CO}_{2}$ and $35 \% \mathrm{~N}_{2}$ ) was provided by Nanfang Hospital Oxygen Center (Guangzhou, China). High glucose Dulbecco's modified Eagle's medium (DMEM, cat\#C11995) and fetal bovine serum (FBS, cat\#10099-141) were purchased from Gibco (Carlsbad, CA, USA). The Lipofectamine 2000 reagent (cat\#11668-019) was purchased from Invitrogen (Carlsbad, CA, USA). The cell counting kit-8 (CCK8) was purchased from Dojindo (Kumamoto, Japan). The ROS kit was purchased from Nanjing Jiancheng Bioengineering Institute (Nanjing, China). The PCR primers were synthesized by Invitrogen Biot echnology Co., Ltd. (Shanghai, China). TRIzol reagent, the PrimeScript RT reagents kit and the SYBR Premix Ex Taq kit was purchased from Takara Bio (Dalian, China).

Cell culture. The EA.hy926 cell line was obtained from the Shanghai Institute Cell Bank (Shanghai, China), and cultured in high-glucose DMEM with $10 \% \mathrm{FBS}$ at $5 \% \mathrm{CO}_{2}$ in a $37^{\circ} \mathrm{C}$ humidified incubator. The EA.hy 926 cells were divided into 4 groups as follows: the air group, the hyperoxia group, the hyperoxia plus PBEF siRNA group and the hyperoxia plus scramble siRNA group. The cells in the air group were cultured for 24,48 and $72 \mathrm{~h}$ in a $5 \% \mathrm{CO}_{2}, 95 \%$ air, $37^{\circ} \mathrm{C}$ incubator. The cells in the hyperoxia group were cultured for 24,48 and $72 \mathrm{~h}$ in the $37^{\circ} \mathrm{C}$ triple gas mixture. The cells in the hyperoxia plus PBEF siRNA group were transfected with PBEF siRNA, and cultured for 24,48 and $72 \mathrm{~h}$ in the $37^{\circ} \mathrm{C}$ triple gas mixture. The cells in the hyperoxia plus scramble siRNA group were transfected with scramble siRNA, and cultured for 24, 48 and $72 \mathrm{~h}$ in the $37^{\circ} \mathrm{C}$ triple gas mixture.

Transient transfection of siRNA. PBEF stealth siRNAs were designed by Ye et al previously (21) (sense sequence, 5'-CCACCCAACA CAAGCAAAGUUUAUU-3' and antisense sequence, 5'-AAUAAAGUUUGGUUGUGUUGGGUGG-3'). To transfect PBEF siRNA into the EA.hy926 cells, the cells were plated for $24 \mathrm{~h}$ in high-glucose DMEM (without antibiotics) prior to transfection, until they reach $60-70 \%$ confluency at the time of transfection. For each transfection in 6-well culture plates, 100 pmol PBEF siRNA were diluted in $250 \mu \mathrm{l}$ Opti-MEM I medium without serum and gently mixed with $5 \mu 1$ Lipofectamine 2000 diluted in $250 \mu 1$ Opti-MEM I medium (Invitrogen). PBEF siRNA and Lipofectamine 2000 complexes were incubated for $15 \mathrm{~min}$ at $37^{\circ} \mathrm{C}$ and the complexes were then added to each well, and the cell culture plates were gently rocked back and forth for uniform mixing. The amount of PBEF stealth siRNA and Lipofectamine 2000 were adjusted according to the different sizes of the cell culture plates. The scramble siRNA were transfected into the EA.hy926 cells in the same manner. The PBEF siRNA and scramble siRNA were synthesized and purified by GenePharma (Shanghai, China). Transfected cells were further incubated at $37^{\circ} \mathrm{C}$ for $24 \mathrm{~h}$ until the intended assays were carried out.

Cell proliferation and viability detection by CCK8. The EA.hy926 cells were plated at a density of $1 \times 10^{4}$ cells/well in a 96-well plate. The cells in the air group were culured for 24 , 48 and $72 \mathrm{~h}$ in a $5 \% \mathrm{CO}_{2}, 95 \%$ air, $37^{\circ} \mathrm{C}$ incubator. The cells
Table I. Primers in quantitative PCR.

\begin{tabular}{ll} 
Gene symbol & \multicolumn{2}{c}{ Primer sequences } \\
\hline PBEF & F: 5'-AAGCTTTTTAGGGCCCTTTG-3' \\
& R: 5'-AGGCCATGTTTTATTTGCTGA-3' \\
IL-8 & F: 5'-AGCAAAATTGAGGCCAAGG-3' \\
& R: 5'-AAACCAAGGCACAGTGGAAC-3' \\
TNF- $\alpha$ & F: 5'-CCTGTGAGGAGGAGGAACAT-3' \\
& R: 5'-GGTTGAGGGTGTCTGAAGGA-3' \\
$\beta$-actin & F: 5'-CATGTACGTTGCTATCCAGGC-3' \\
& R: 5'-CTCCTTAATGTCACGCACGAT-3'
\end{tabular}

PBEF, pre-B cell colony-enhancing factor; IL-8, interleukin-8; TNF- $\alpha$, tumor necrosis factor- $\alpha$; F, forward; R, reverse.

in the hyperoxia group were cultured for 24,48 and $72 \mathrm{~h}$ in the $37^{\circ} \mathrm{C}$ triple gas mixture. The cells in the hyperoxia plus PBEF siRNA group were transfected with PBEF siRNA in advance, and then plated at a density of $1 \times 10^{4}$ cells/well in a 96-well plate, followed by culture for 24,48 and $72 \mathrm{~h}$ in the $37^{\circ} \mathrm{C}$ triple gas mixture. A total of $10 \mu 1$ of CCK 8 (Dojindo) was added to the cells, and the viability of the cells was measured at $450 \mathrm{~nm}$ using an MD5 microplate reader (SpectraMax M5; Molecular Devices Company, Sunnyvale, CA, USA) according to the manufacturer's instructions.

Assessment of ROS generation. The ROS activities were measured using a specific assay kit (Nanjing Jiancheng Bioengineering Institute, Nanjing, China) according to the manufacturer's instructions. The cells were washed twice with phosphate-buffered saline (PBS), and $1 \mathrm{ml} \mathrm{H}_{2} \mathrm{O}_{2}(100 \mu \mathrm{M})$ was added to the cells in the air group as the positive control, and $1 \mathrm{ml} \mathrm{2',7'-dichloro-fluorescein-diacetate} \mathrm{(DCFH-DA;} 10 \mu \mathrm{M}$ ) was added to the cells in the air group, the hyperoxia group and the hyperoxia plus PBEF siRNA group. The cells were then incubated at $37^{\circ} \mathrm{C}$ for $30 \mathrm{~min}$. Following the removal of the mixture from the Petri dish, the cells were washed twice with PBS. The fluorescence was measured using an MD5 microplate reader.

Quantitative PCR ( $q P C R)$. Total RNA was isolated from the cells using TRIzol reagent (Takara Bio) following the manufacturer's instructions. Single-strand cDNA was synthesized using $2 \mathrm{mg}$ of total RNA by the reverse transcription (RT) reaction using the PrimeScript RT reagents kit (Takara Bio). Real-time (quantitative) PCR was conducted using an Applied Biosystems 7500 real-time PCR system according to the instructions provided with the SYBR Premix Ex Taq kit (Takara Bio). The thermal cycling parameters consisted of $95^{\circ} \mathrm{C}$ for $30 \mathrm{sec}$, followed by 40 cycles of $95^{\circ} \mathrm{C}$ for $5 \mathrm{sec}$ and $60^{\circ} \mathrm{C}$ for $34 \mathrm{sec}$. Relative expression values were normalized using an internal $\beta$-actin control. The fold-change of relative gene expression levels was calculated using the $2^{-\Delta \Delta C q}$ method, and the primer sequences are listed in Table I.

Western blot analysis. Total protein was extracted from the cells with lysis buffer using the Total Protein Extraction 


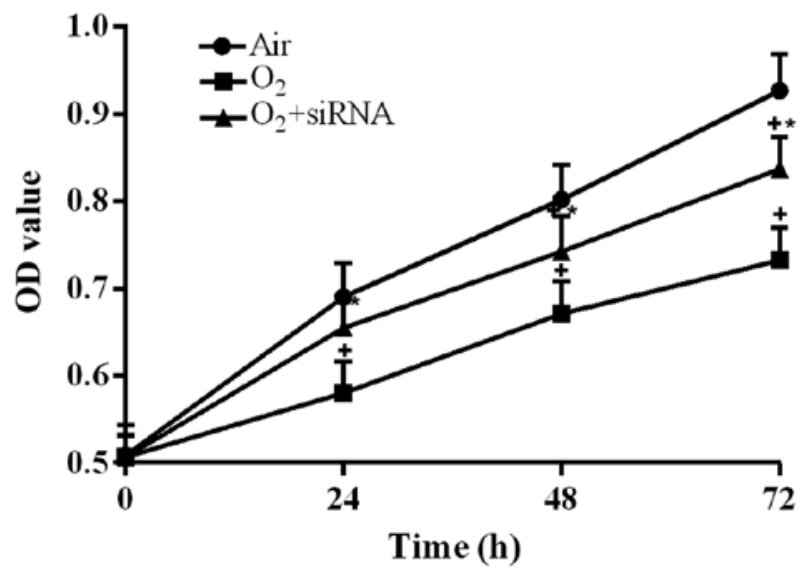

Figure 1 . The viability of EA.hy926 cells in each group. The cells were incubated for 24, 48 and $72 \mathrm{~h}$, and cell viability was examined by cell counting kit-8 (CCK8) assay. Values are expressed as the means $\pm \mathrm{SD}(\mathrm{n}=8) .{ }^{+} \mathrm{P}<0.05$ vs. air group; ${ }^{*} \mathrm{P}<0.05$ vs. $\mathrm{O}_{2}$ group. $\mathrm{O}_{2}$, hyperoxia; $\mathrm{O}_{2}+$ siRNA, hyperoxia plus pre-B cell colony-enhancing factor (PBEF) siRNA.

reagent kit (KeyGen, Nanjing, China). The protein concentration was measured by BCA protein assay (Keygen). Protein extract samples (30 $\mu \mathrm{g} /$ lane) were analyzed by $10 \%$ sodium dodecyl sulfate-polyacrylamide gel electrophoresis (SDS-PAGE) and transferred onto PVDF membranes (Millipore, Bedford, MA USA). The membranes were subsequently blocked with $5 \%$ non-fat milk solution, and incubated with monoclonal anti- $\beta$-actin (Cat. no. AF0003; Bytotime Biotechnology, Jiangsu, China), anti-PBEF (Cat. no. sc-67020; Santa Cruz Biotechnology, Inc., Dallas, TX, USA), anti-TNF- $\alpha$ (Cat. no. BS6000) and anti-IL-8 (Cat. no. BS7145) (both from Bioworld Technology, Inc., St. Louis Park, MN, USA) antibodies, diluted at 1:1,000. Anti-rabbit $(\mathrm{H}+\mathrm{L}) \mathrm{HRP}$ diluted at 1:2,000 was used as the secondary antibody with SuperSignal West Pico Chemiluminescent Substrate (Thermo Fisher Scientific Inc., Rockford, IL, USA) for detection. The intensities of the protein bands were analyzed using Quantity One software (Bio-Rad Laboratories, Hercules, CA, USA).

Statistical analysis. The results are presented as the means \pm SD. Statistical analysis was analyzed using SPSS 13.0 statistical software (SPSS, Inc., Chicago, IL, USA). Stimulated samples were compared with the controls by an unpaired Student's t-test. One-way analysis of variance (ANOVA) was used for multiplegroup comparisons. A value of $\mathrm{P}<0.05$ was considered to indicate a statistically significant difference.

\section{Results}

Cell viability. The cells in the air group were cultured for 24,48 and $72 \mathrm{~h}$ in a $5 \% \mathrm{CO}_{2}, 95 \%$ air, $37^{\circ} \mathrm{C}$ incubator, while the cells in the hyperoxia group and the hyperoxia plus PBEF siRNA group were cultured for 24,48 and $72 \mathrm{~h}$ in the $37^{\circ} \mathrm{C}$ triple gas mixture. The results of CCK8 assay revealed that hyperoxia significantly decreased cell viability by $16 \%$ after $24 \mathrm{~h}, 17 \%$ after $48 \mathrm{~h}$ and $21 \%$ after $72 \mathrm{~h}$ compared with the air group, while cell viability in the hyperoxia plus PBEF siRNA group was significantly higher than that of the hyperoxia group $(\mathrm{P}<0.05$; Fig. 1). These

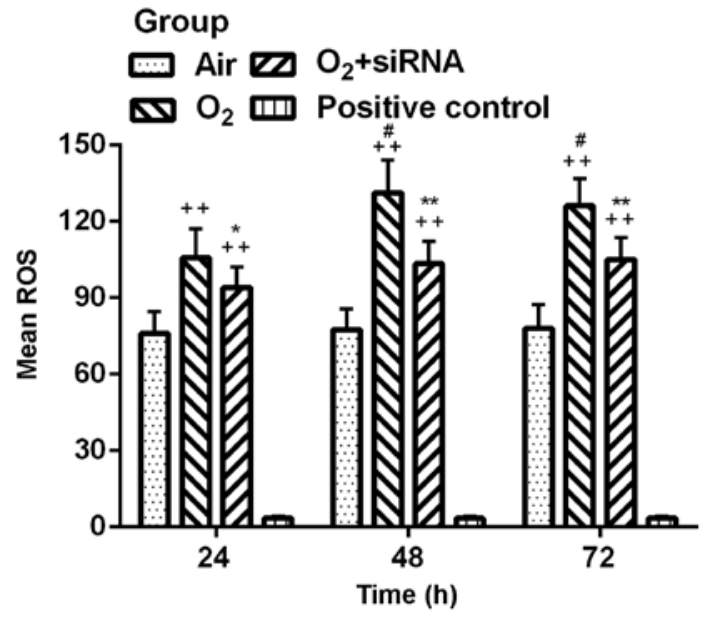

Figure 2. The reactive oxygen species (ROS) levels in EA.hy926 cells in each group. Values are the means $\pm \mathrm{SD}(\mathrm{n}=8)$. ${ }^{*} \mathrm{P}<0.05$, significantly different from the time-matched $\mathrm{O}_{2}$ group; ${ }^{* *} \mathrm{P}<0.01$, significantly different from the timematched $\mathrm{O}_{2}$ group; ${ }^{++} \mathrm{P}<0.01$, significantly different from the time-matched air group; ${ }^{~} \mathrm{P}<0.01$, significantly different from the $24 \mathrm{~h}$ time point. $\mathrm{O}_{2}$, hyperoxia; $\mathrm{O}_{2}+$ siRNA, hyperoxia plus pre-B cell colony-enhancing factor (PBEF) siRNA. Positive control, $1 \mathrm{ml} \mathrm{H}_{2} \mathrm{O}_{2}(100 \mu \mathrm{M})$ was added to the cells of the air group.

results indicate that PBEF silencing suppresses cell damage induced by hyperoxia.

Assessment of ROS levels. As shown in Fig. 2, the average ROS levels were $77.156 \pm 8.465,121.077 \pm 15.795$ and $100.857 \pm 9.567$ in the air, hyperoxia and hyperoxia plus PBEF siRNA groups. The ROS levels in the hyperoxia group and the hyperoxia plus PBEF siRNA group were significantly higher than those in the air group at 24, 48 and $72 \mathrm{~h}(\mathrm{P}<0.01)$, while the ROS levels in the hyperoxia plus PBEF siRNA group was significantly lower than those in the hyperoxia group $(\mathrm{P}<0.01)$. The ROS levels in the hyperoxia group at 48 and $72 \mathrm{~h}$ were significantly higher compared to those at $24 \mathrm{~h}(\mathrm{P}<0.01)$. The results demonstrate that the silencing of PBEF reduces the ROS levels induced by hyperoxia in the EA.hy926 cells.

Changes in the MRNA expression of PBEF, IL- 8 and TNF- $\alpha$ detected by $q P C R$. As shown in Fig. 3, qPCR analysis revealed that the PBEF, IL-8, TNF- $\alpha$ mRNA expression levels in the hyperoxia group were significantly higher than those in the air group at each time point $(\mathrm{P}<0.01)$; however, these mRNA expression levels were significantly decreased in the hyperoxia plus PBEF siRNA group compared with the hyperoxia group at each time point $(\mathrm{P}<0.01)$. In the hyperoxia plus $\mathrm{PBEF}$ siRNA group, it was shown that PBEF siRNA knocked down PBEF mRNA expression in the EA.hy926 cells ( $>65 \%$ decrease, $\mathrm{P}<0.01$; Fig. 3). Scramble siRNA alone had no effect on PBEF expression. The TNF- $\alpha$ and PBEF gene expression levels in the hyperoxia group and the hyperoxia plus PBEF siRNA group at 48 and $72 \mathrm{~h}$ were significantly higher than the levels at $24 \mathrm{~h}(\mathrm{P}<0.01)$. The PBEF gene expression levels in the hyperoxia group and the hyperoxia plus PBEF siRNA group at $72 \mathrm{~h}$ were significantly higher than the levels at 24 and $48 \mathrm{~h}(\mathrm{P}<0.01)$. No significantly differences were observed between the hyperoxia group and the hyperoxia plus scramble siRNA group at any time point. These results provided evidence that the mRNA 


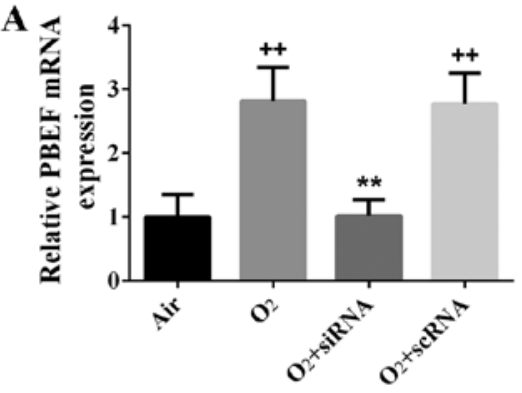

$24 \mathrm{~h}$

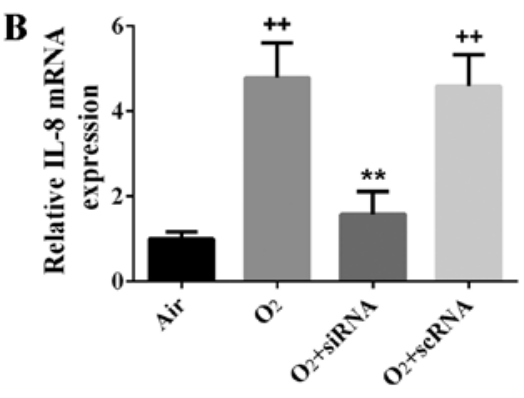

24 h

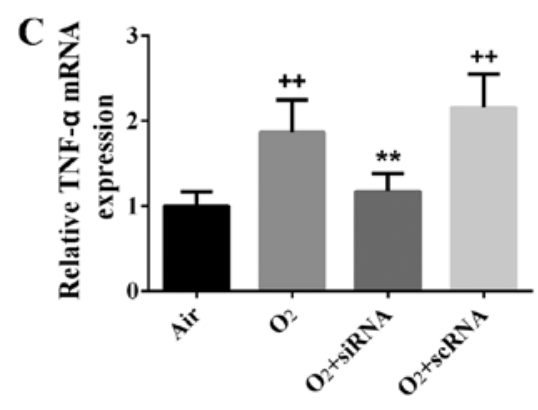

24 h

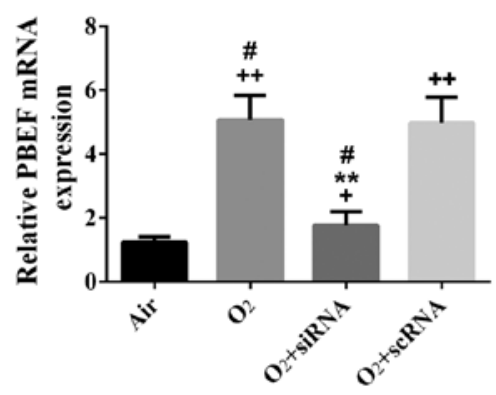

$48 \mathrm{~h}$

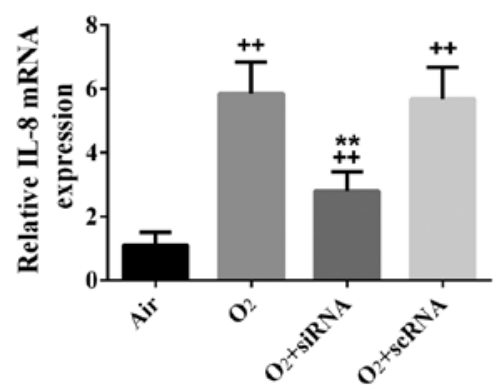

$48 \mathrm{~h}$

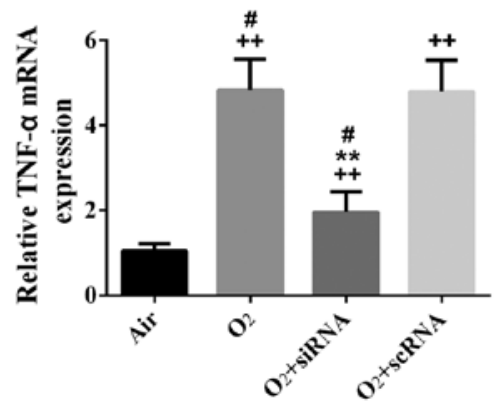

$48 \mathrm{~h}$

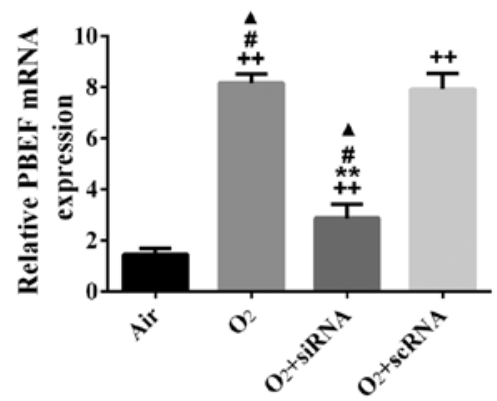

72 h

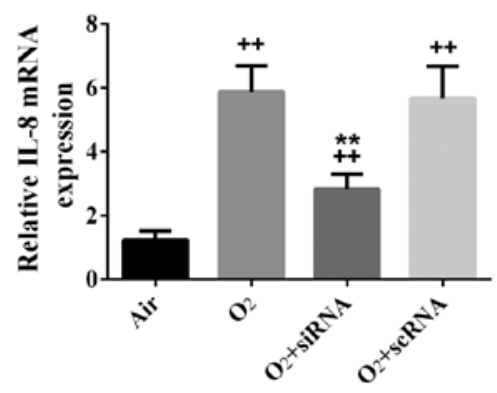

$72 \mathrm{~h}$

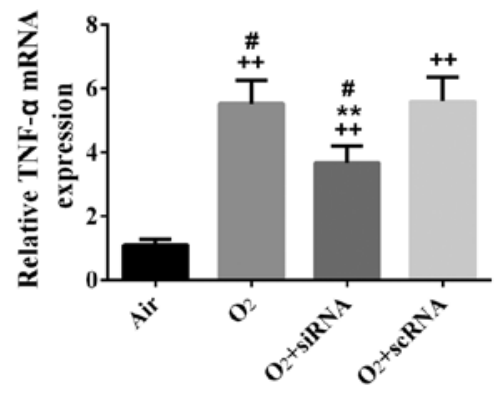

72 h

Figure 3. Induction and inhibition of the mRNA expression of inflammatory cytokines in EA.hy926 cells. mRNA expression was measured using the $2^{-\Delta \Delta \mathrm{Ct}}$ method. (A) Induction and inhibition of pre-B cell colony-enhancing factor (PBEF) mRNA expression in EA.hy926 cells. (B) Induction and inhibition of interleukin-8 (IL-8) mRNA expression in EA.hy926 cells. (C) Induction and inhibition of tumor necrosis factor- $\alpha$ (TNF- $\alpha$ ) mRNA expression in EA.hy926 cells. Values are the means $\pm \mathrm{SD} .{ }^{* *} \mathrm{P}<0.01$, significantly different from the time-matched $\mathrm{O}_{2}$ group; ${ }^{++} \mathrm{P}<0.01$, significantly different from the time-matched air group. ${ }^{\#} \mathrm{P}<0.01$, significantly different from the $24 \mathrm{~h}$ time point. ${ }^{\wedge} \mathrm{P}<0.01$, significantly different from the $48 \mathrm{~h}$ time point. $\mathrm{O}_{2}$, hyperoxia; $\mathrm{O}_{2}+$ siRNA, hyperoxia plus PBEF siRNA; $\mathrm{O}_{2}+$ scRNA, hyperoxia plus scramble siRNA.

expression of PBEF was significantly increased in the hyperoxia group, and the silencing of PBEF inhibited IL- 8 and TNF- $\alpha$ mRNA expression which was induced by hyperoxia.

Detection of PBEF, IL- 8 and TNF- $\alpha$ protein expression in EA.hy926 cells by western blot analysis. The results from western blot analysis revealed that the protein expression of PBEF, IL- 8 and TNF- $\alpha$ in the hyperoxia group was increased compared with the air group $(\mathrm{P}<0.01)$, while the protein expression of PBEF, IL-8, TNF- $\alpha$ in the hyperoxia plus PBEF siRNA group was significantly lower than that in the hyperoxia group $(\mathrm{P}<0.01$; Fig. 4). In the hyperoxia plus PBEF siRNA group, PBEF siRNA knocked down PBEF protein expression in the EA.hy926 cells ( $>65 \%$ decrease, $\mathrm{P}<0.01$; Fig . 4A and B). Scramble siRNA alone had no effect on PBEF expression compared with the hyperoxia group. The TNF- $\alpha$ and PBEF protein expression levels in the hyperoxia group and the hyperoxia plus PBEF siRNA group at 48 and $72 \mathrm{~h}$ were significantly higher than the levels at $24 \mathrm{~h}(\mathrm{P}<0.01)$. No significant differences were observed between the hyperoxia group and the hyperoxia plus scrabmle siRNA group at any time point (Fig. 4). These results demonstrated that hyperoxia increased the expression of inflammatory cytokines in the EA.hy926 cells and the knockdown of PBEF expression attenuated the promoting effects of hyperoxia on cytokine expression, indicating that PBEF is critically involved in the regulation of the inflammatory response to hyperoxia in EA.hy926 cells.

\section{Discussion}

Our findings demonstrate that the levels of the pro-inflammatory cytokines, PBEF, TNF- $\alpha$ and IL-8, as well as the ROS level were increased in the EA.hy926 cells following exposure to hyperoxia, and the knockdown of PBEF using siRNA in 
A

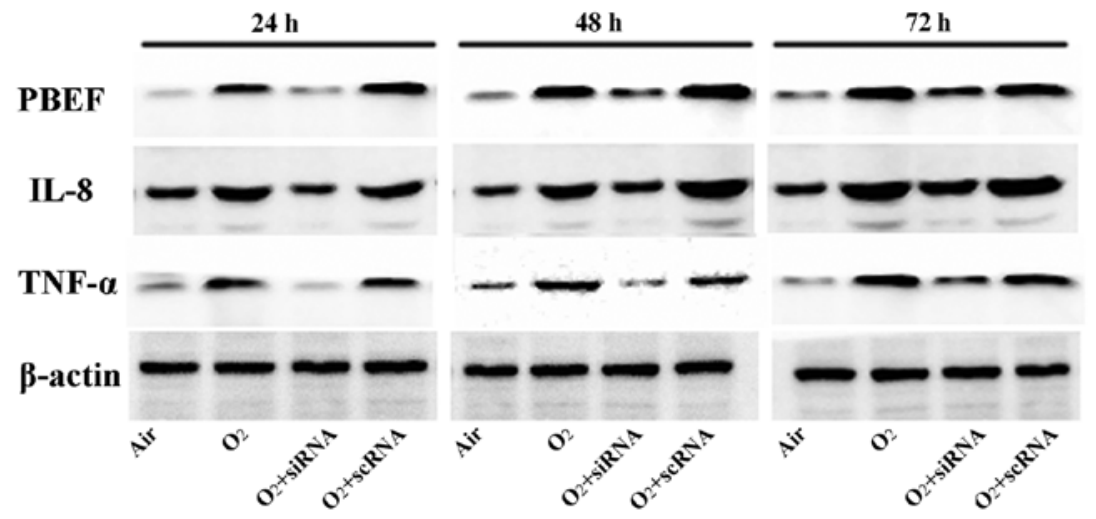

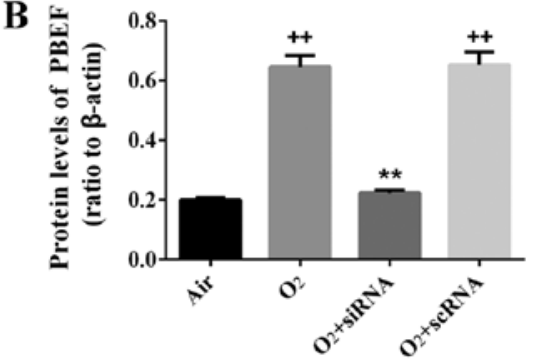

24 h

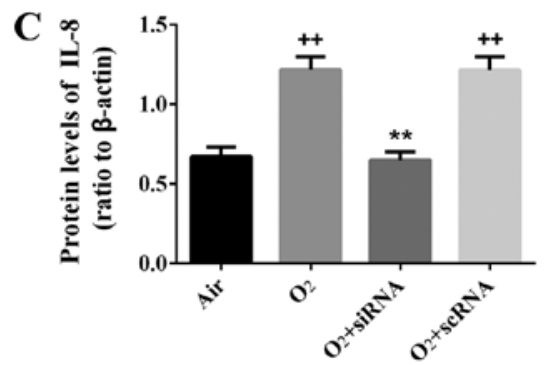

$24 \mathrm{~h}$

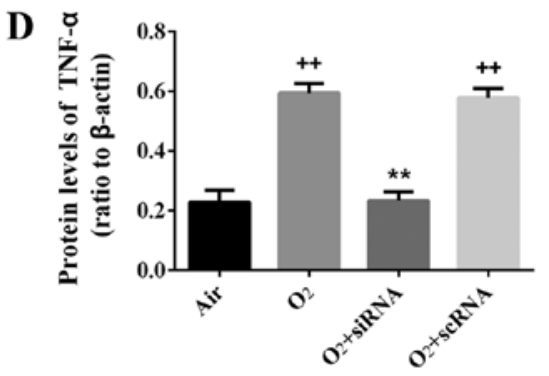

24 h

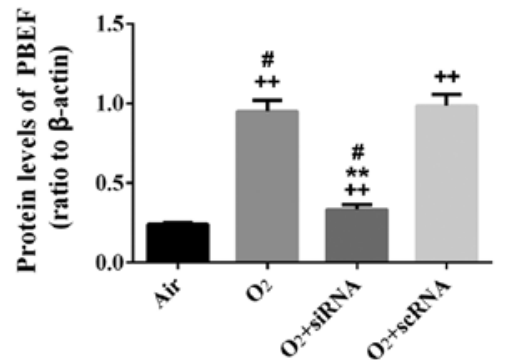

$48 \mathrm{~h}$

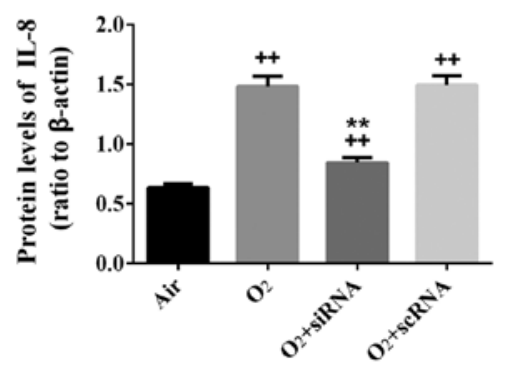

$48 \mathrm{~h}$

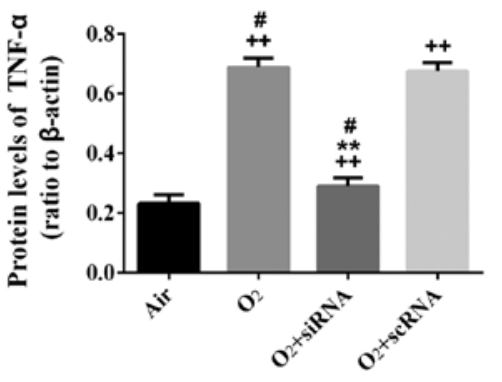

$48 \mathrm{~h}$

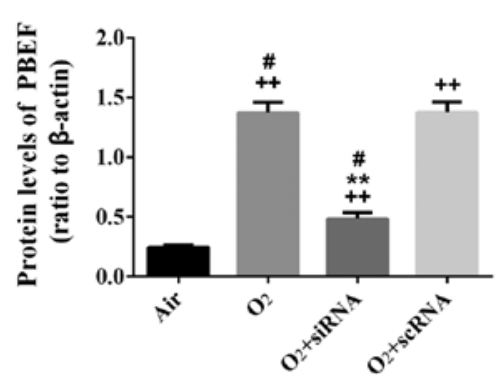

72 h

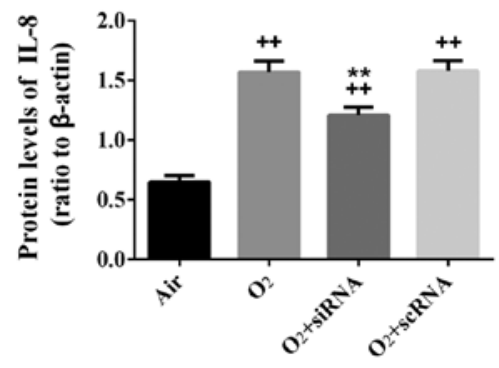

72 h

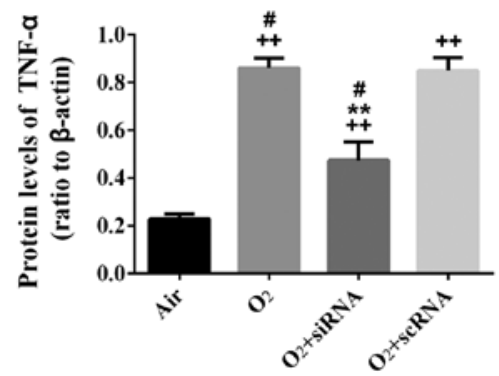

72 h

Figure 4. Induction and inhibition of the protein expression of inflammatory cytokines in EA.hy926 cells. (A) The protein expression of pre-B cell colonyenhancing factor (PBEF), interleukin-8 (IL-8), tumor necrosis factor- $\alpha$ (TNF- $\alpha$ ) in EA.hy 926 cells from each group identified by western blot analysis. $\beta$-actin was used to verify equivalent loading. (B) Induction and inhibition of PBEF protein expression in EA.hy 926 cells. (C) Induction and inhibition of IL-8 protein expression in EA.hy926 cells. (D) Induction and inhibition of TNF- $\alpha$ protein expression in EA.hy926 cells. Values are means \pm SD. ${ }^{* *} \mathrm{P}<0.01$, significantly different from the time-matched $\mathrm{O}_{2}$ group; ${ }^{++} \mathrm{P}<0.01$, significantly different from the time-matched air group. ${ }^{\text {}} \mathrm{P}<0.01$, significantly different from the $24 \mathrm{~h}$ time point. $\mathrm{O}_{2}$, hyperoxia; $\mathrm{O}_{2}+\mathrm{siRNA}$, hyperoxia plus PBEF siRNA; $\mathrm{O}_{2}+$ scRNA, hyperoxia plus scramble siRNA.

the EA.hy926 cells significantly inhibited the expression of the cytokines mentioned above, as well as the ROS levels. Furthermore, hyperoxia induced the EA.hy926 cell apoptosis, and the silencing of PBEF suppressed the cell growth inhibition induced by hyperoxia. These results provide new insight into the role of PBEF in the inflammatory pathways and functional abnormalities associated with BPD.

There is considerable experimental and clinical evidence that pro-inflammatory cytokines play a crucial role in the pathogenesis of BPD, including IL-6, IL-8, IL-10, TNF- $\alpha$ 
and ICAM-1 $(22,23)$. TNF- $\alpha$ and IL-8 are among important early mediators of BPD. TNF- $\alpha$ has been reported to involved in many inflammatory disease, such as acute respiratory distress syndrome, BPD and chronic obstructive pulmonary disease $(24,25)$. It has been shown that TNF- $\alpha$ is present in increased amounts in the bronchoalveolar lavage fluid (BALF) of rats with BPD (23). The role of TNF- $\alpha$ in pulmonary pathophysiology has been investigated by Robriquet $e t$ al, including the activation of oxidative stress and the induction of cellular inflammatory reactions (26).

Among the pro-inflammatory cytokines, IL-8 is regarded as another important mediator in the pathogenesis of BPD (22). In fact, IL-8 was first purified and molecularly cloned by Matsushima et al in 1988 as a neutrophil chemotactic factor from lipopolysaccharide-stimulated human mononuclear cell supernatants (27). IL-8 has been reported to induce actin fiber formation and intercellular gap formation in endothelial cells (28). Dunican et al demonstrated that the TNF- $\alpha$ mediated IL-8 production attenuated neutrophil apoptosis and thus potentially prolonged neutrophil migration into the lungs and damage to lung tissues (29). It has also been demonstrated that PBEF may play a vital role in the augmentation of pulmonary epithelial cell IL-8 expression induced by TNF- $\alpha$ (10).

The data of the present study indicate that hyperoxia significantly induces PBEF, IL-8 and TNF- $\alpha$ expression at both the mRNA and protein level in EA.hy926 cells (Figs. 3 and 4), suggesting that PBEF, IL-8 and TNF- $\alpha$ are involved in the inflammatory process during the pathogenesis of BPD. The knockdown of PBEF expression by PBEF siRNA markedly blunted TNF- $\alpha$ and IL-8 expression in the EA.hy 926 cells. These results support the concept that PBEF may be an inflammatory signal transducer of TNF- $\alpha$ and IL-8 or other inflammatory cytokines. These conclusions can be demonstrated by evidence in non-lung tissue studies. Jia et al reported that the silencing of PBEF prevented the suppression of neutrophil apoptosis caused by TNF- $\alpha$, IL- 8 and other mediators (7). Furthermore, it has been suggest that the treatment of amnion-like epithelial cells with recombinant human PBEF significantly increased IL-8 and IL-6 expression (30). Taken together, these data indicate that PBEF may play a crucial role as an inflammatory cytokine during the pathogenesis of BPD.

There is increasing evidence to indicate that oxidative stress is one of the most important events in the development of BPD $(31,32)$. It has been reported that oxidative stress plays an important role in endothelial dysfunction and vascular injury (33). Oxidative stress is caused by an imbalance between ROS generation and the antioxidant defense system (34). The balance is likely to be broken when the overexpression or inadequate clearance of ROS becomes uncontrollable, resulting in severe damage to DNA, protein and lipids (34-36). ROS influence a number of cellular responses by affecting several intracellular signaling cascades, which contribute to altered inflammation and vascular remodeling (37). The premature infant is particularly sensitive to ROS-induced damage due to deficient antioxidant stores at birth, as well as impaired upregulation in oxidant stress response. Therefore, the premature infant is at high risk of the development of ROS-induced diseases, such as BPD, necrotizing enterocolitis, periventricular leukomalacia and retinopathy of prematurity (34). In this study, the ROS levels in the hyperoxia group were significantly higher than those in the air group $(\mathrm{P}<0.01)$, while the inhibition of PBEF expression by PBEF siRNA significantly blunted hyperoxia-induced ROS production compared with the hyperoxia group (Fig. 2). These results indicated that PBEF affects intracellular oxidative stress. Kim et al demonstrated that PBEF stimulated ROS generation in endothelial cells, and antioxidants blocked PBEF-induced NF- $\mathrm{KB}$ activation and CAM expression (11). In addition, the interacting partners of PBEF in oxidative stress have been identified, including NADH dehydrogenase subunit 1 (ND1), ferritin and interferon-induced transmembrane 3 (IFITM3) (38). Taken together, the abovementioned data suggest that the interaction between PBEF and oxidative stress may be a potential mechanism in the pathogenesis of BPD.

It is well known that apoptosis is critical for normal lung development and function $(39,40)$ and plays a key role in remodeling of lung tissue by clearing excess epithelial and mesenchymal cells following injury $(41,42)$. However, abnormal apoptotic activity may contribute to the pathophysiology of a number of lung diseases, including BPD. Kazzaz et al reported that apoptosis was clearly induced in the lungs of mice exposed to hyperoxia (43). Normally, the lung alveolar epithelium forms a tight barrier to restrict the movement of proteins and liquid from the interstitium into the alveolar spaces. If the alveolar epithelium is damaged, both the permeability of endothelial cells and epithelial cells changes, and this may lead to major alveolar flooding with high-molecular-weight proteins, with prolonged changes in gas exchange $(44,45)$. In this study, the viability of the cells in the hyperoxia group was significantly decreased compared with that of the cells in the air group, and the induction of apoptosis by hyperoxia was observed in the cultured cells. Exposure to high oxygen concentrations leads to oxidative stress and activates the inflammatory response, which may cause cell death. However, the knockdown of PBEF increased cell viability in the hyperoxia plus PBEF siRNA group. The exact mechanisms behind this effect remain unknown; however, the silencing of PBEF attenuated oxidative stress and the inflammatory response, and this may suppress the cell damage caused by hyperoxia. Recently, a study demonstrated that PBEF was involved in the process of microvascular endothelial cell apoptosis through the Fas/FasL-mediated extrinsic apoptotic pathway (46). Ming et al demonstrated that PBEF promoted the apoptosis of pulmonary microvascular endothelial cells and regulated the expression of inflammatory factors and the expression of aquaporin 1 (AQP1) through the MAPK pathways (47). These results indicate that the inhibition PBEF signaling may be a potential strategy with which to attenuate endothelial cell apoptosis.

In conclusion, this study demonstrated that hyperoxia significantly induced PBEF, IL- 8 and TNF- $\alpha$ expression at both the mRNA and protein level in EA.hy926 cells. The knockdown of PBEF expression by PBEF siRNA significantly blunted TNF- $\alpha$ and IL- 8 mRNA and protein production, and attenuated the hyperoxia-induced increase in ROS levels in EA.hy 926 cells. Since inflammation and oxidative stress are the hallmarks of the pathogenesis of BPD, these results suggest that PBEF plays a key role as a pro-inflammatory cytokine in the dysregulation of alveolar epithelial cell barriers in the development of BPD. These results lend further support to 
the potential of PBEF to serve as a diagnostic and therapeutic target in future studies of BPD.

\section{Acknowledgements}

The present study was supported by the Guangdong Province Science and Technology Plan Project (00465510217884051 to W.M.H.)

\section{References}

1. Fernandez-Gonzalez A, Alex MS, Liu X and Kourembanas S: Vasculoprotective effects of heme oxygenase-1 in a murine model of hyperoxia-induced bronchopulmonary dysplasia. Am J Physiol Lung Cell Mol Physiol 302: L775-L784, 2012.

2. Merritt TA, Deming DD and Boynton BR: The 'new' bronchopulmonary dysplasia: Challenges and commentary. Semin Fetal Neonatal Med 14: 345-357, 2009.

3. Joung KE, Kim HS, Lee J, Shim GH, Choi CW, Kim EK, Kim BI and Choi JH: Correlation of urinary inflammatory and oxidative stress markers in very low birth weight infants with subsequent development of bronchopulmonary dysplasia. Free Radic Res 45 : 1024-1032, 2011.

4. Gien J and Kinsella JP: Pathogenesis and treatment of bronchopulmonary dysplasia. Curr Opin Pediatr 23: 305-313, 2011.

5. Köksal N, Kayik B, Çetinkaya M, Özkan H, Budak F, Kiliç S Canitez Y and Oral B: Value of serum and bronchoalveolar fluid lavage pro- and anti-inflammatory cytokine levels for predicting bronchopulmonary dysplasia in premature infants. Eur Cytokine Netw 23: 29-35, 2012

6. Samal B, Sun Y, Stearns G, Xie C, Suggs S and McNiece I: Cloning and characterization of the cDNA encoding a novel human pre-B-cell colony-enhancing factor. Mol Cell Biol 14 1431-1437, 1994.

7. Jia SH, Li Y, Parodo J, Kapus A, Fan L, Rotstein OD and Marshall JC: Pre-B cell colony-enhancing factor inhibits neutrophil apoptosis in experimental inflammation and clinical sepsis. J Clin Invest 113: 1318-1327, 2004.

8. Moschen AR, Kaser A, Enrich B, Mosheimer B, Theurl M, Niederegger $\mathrm{H}$ and Tilg $\mathrm{H}$ : Visfatin, an adipocytokine with proinflammatory and immunomodulating properties. J Immunol 178 1748-1758, 2007.

9. Ye SQ, Simon BA, Maloney JP, Zambelli-Weiner A, Gao L, Grant A, Easley RB, McVerry BJ, Tuder RM, Standiford T, et al: Pre-B-Cell colony-enhancing factor as a potential novel biomarker in acute lung injury. Am J Resp Crit Care 171: 361-370, 2005.

10. Li H, Liu P, Cepeda J, Fang D, Easley RB, Simon BA, Zhang L and Ye S: Augmentation of pulmonary epithelial cell IL-8 expression and permeability by pre-B-cell colony enhancing factor. J Inflamm (Lond) 5: 15, 2008.

11. Kim SR, Bae YH, Bae SK, Choi KS, Yoon KH, Koo TH, Jang HO, Yun I, Kim KW, Kwon YG, et al: Visfatin enhances ICAM-1 and VCAM-1 expression through ROS-dependent NF- $\mathrm{BB}$ activation in endothelial cells. Biochim Biophys Acta 1783: 886-895, 2008.

12. Otero M, Lago R, Gomez R, Lago F, Dieguez C, Gomez-Reino JJ and Gualillo O: Changes in plasma levels of fat-derived hormones adiponectin, leptin, resistin and visfatin in patients with rheumatoid arthritis. Ann Rheum Dis 65: 1198-1201, 2006.

13. Fan Y, Meng S, Wang Y, Cao J and Wang C: Visfatin/PBEF/ Nampt induces EMMPRIN and MMP-9 production in macrophages via the NAMPT-MAPK (p38, ERK1/2)-NF- $\kappa$ B signaling pathway. Int J Mol Med 27: 607-615, 2011.

14. Edgell CJ, McDonald CC and Graham JB: Permanent cell line expressing human factor VIII-related antigen established by hybridization. Proc Natl Acad Sci USA 80: 3734-3737, 1983.

15. Edgell CJ, Haizlip JE, Bagnell CR, Packenham JP, Harrison P, Wilbourn B and Madden VJ: Endothelium specific Weibel-Palade bodies in a continuous human cell line, EA.hy926. In Vitro Cell Dev Biol 26: 1167-1172, 1990.

16. Xu D, Perez RE, Ekekezie II, Navarro A and Truog WE: Epidermal growth factor-like domain 7 protects endothelial cells from hyperoxia-induced cell death. Am J Physiol Lung Cell Mol Physiol 294: L17-L23, 2008.
17. Thornhill MH, Li J and Haskard DO: Leucocyte endothelial cell adhesion: A study comparing human umbilical vein endothelial cells and the endothelial cell line EA-hy-926. Scand J Immunol 38: 279-286, 1993.

18. Song YH, Neumeister MW, Mowlavi A and Suchy H: Tumor necrosis factor-alpha and lipopolysaccharides induce differentially interleukin 8 and growth related oncogene-alpha expression in human endothelial cell line EA.hy926. Ann Plast Surg 45: 681-683, 2000

19. Aranda E and Owen GI: A semi-quantitative assay to screen for angiogenic compounds and compounds with angiogenic potential using the EA.hy926 endothelial cell line. Biol Res 42: 377-389, 2009.

20. Cai W, Li Y, Yi Q, Xie F, Du B, Feng L and Qiu L: Total saponins from Albizia julibrissin inhibit vascular endothelial growth factor-mediated angiogenesis in vitro and in vivo. Mol Med Rep 11: 3405-3413, 2015.

21. Ye SQ,Zhang LQ, Adyshev D, Usatyuk PV, Garcia AN, Lavoie TL, Verin AD, Natarajan V and Garcia JGN: Pre-B-cell-colonyenhancing factor is critically involved in thrombin-induced lung endothelial cell barrier dysregulation. Microvasc Res 70: 142-151, 2005.

22. Huang WM, Liang YQ, Tang LJ, Ding Y and Wang XH: Antioxidant and anti-inflammatory effects of Astragalus polysaccharide on EA.hy926 cells. Exp Ther Med 6: 199-203, 2013.

23. Wang X, Jia H, Deng L and Huang W: Astragalus polysaccharides mediated preventive effects on bronchopulmonary dysplasia in rats. Pediatr Res 76: 347-354, 2014.

24. Bose CL, Dammann CE and Laughon MM: Bronchopulmonary dysplasia and inflammatory biomarkers in the premature neonate. Arch Dis Child Fetal Neonatal Ed 93: F455-F461, 2008.

25. SpeerCP: Chorioamnionitis, postnatal factors and proinflammatory response in the pathogenetic sequence of bronchopulmonary dysplasia. Neonatology 95: 353-361, 2009.

26. Robriquet L, Collet $\mathrm{F}$, Tournoys $\mathrm{A}$, Prangère $\mathrm{T}$, Nevière $\mathrm{R}$, Fourrier $\mathrm{F}$ and Guery BP: Intravenous administration of activated protein $\mathrm{C}$ in pseudomonas-induced lung injury: Impact on lung fluid balance and the inflammatory response. Respir Res 7: 41, 2006.

27. Matsushima K1, Morishita K, Yoshimura T,Lavu S, Kobayashi Y, Lew W, Appella E, Kung HF, Leonard EJ and Oppenheim JJ: Molecular cloning of a human monocyte-derived neutrophil chemotactic factor (MDNCF) and the induction of MDNCF mRNA by interleukin 1 and tumor necrosis factor. J Exp Med 167: 1883-1893, 1988.

28. Schraufstatter IU, Chung J and Burger M: IL-8 activates endothelial cell CXCR1 and CXCR2 through Rho and Rac signaling pathways. Am J Physiol Lung Cell Mol Physiol 280: L1094-L1103, 2001.

29. Dunican AL, Leuenroth SJ, Grutkoski P, Ayala A and Simms HH: TNFalpha-induced suppression of PMN apoptosis is mediated through interleukin-8 production. Shock 14: 284-288, 288-289, 2000.

30. Ognjanovic S and Bryant-Greenwood GD: Pre-B-cell colonyenhancing factor, a novel cytokine of human fetal membranes. Am J Obstet Gynecol 187: 1051-1058, 2002.

31. Sampath V, Garland JS, Helbling D, Dimmock D, Mulrooney NP, Simpson PM, Murray JC and Dagle JM: Antioxidant response genes sequence variants and BPD susceptibility in VLBW infants. Pediatr Res 77: 477-483, 2015.

32. Jin L, Yang H, Fu J, Xue X, Yao L and Qiao L: Association between oxidative DNA damage and the expression of 8-oxoguanine DNA glycosylase 1 in lung epithelial cells of neonatal rats exposed to hyperoxia. Mol Med Rep 11: 4079-4086, 2015.

33. Lum $\mathrm{H}$ and Roebuck KA: Oxidant stress and endothelial cell dysfunction. Am J Physiol Cell Physiol 280: C719-C741, 2001.

34. Lee JW and Davis JM: Future applications of antioxidants in premature infants. Curr Opin Pediatr 23: 161-166, 2011.

35. Martindale JL and Holbrook NJ: Cellular response to oxidative stress: Signaling for suicide and survival. J Cell Physiol 192: $1-15,2002$.

36. Welty SE: Is there a role for antioxidant therapy in bronchopulmonary dysplasia? J Nutr 131: 947S-950S, 2001.

37. Fortuno A, San JG, Moreno MU, Diez J and Zalba G: Oxidative stress and vascular remodelling. Exp Physiol 90: 457-462, 2005.

38. Zhang LQ, Adyshev DM, Singleton P, Li H, Cepeda J, Huang SY, Zou X, Verin AD, Tu J, Garcia JGN, et al: Interactions between PBEF and oxidative stress proteins - A potential new mechanism underlying PBEF in the pathogenesis of acute lung injury. FEBS Lett 582: 1802-1808, 2008 . 
39. Kresch MJ, Christian C, Wu F and Hussain N: Ontogeny of apoptosis during lung development. Pediatr Res 43: 426-431, 1998.

40. Kroon AA, DelRiccio V, Tseu I, Kavanagh BP and Post M: Mechanical ventilation-induced apoptosis in newborn rat lung is mediated via FasL/Fas pathway. AJP Lung Cell Mol Physiol 305: L795-L804, 2013.

41. Sherrill DL, Camilli A and Lebowitz MD: On the temporal relationships between lung function and somatic growth. Am Rev Respir Dis 140: 638-644, 1989.

42. Polunovsky VA, Chen B, Henke C, Snover D, Wendt C, Ingbar DH and Bitterman PB: Role of mesenchymal cell death in lung remodeling after injury. J Clin Invest 92: 388-397, 1993.

43. Kazzaz JA, Xu J, Palaia TA, Mantell L, Fein AM and Horowitz S: Cellular oxygen toxicity. Oxidant injury without apoptosis. J Biol Chem 271: 15182-15186, 1996.
44. Liener UC, Bruckner UB, Knoferl MW, Steinbach G, Kinzl L and Gebhard F: Chemokine activation within 24 hours after blunt accident trauma. Shock 17: 169-172, 2002.

45. May M, Strobel P, Preisshofen T, Seidenspinner S, Marx A and Speer CP: Apoptosis and proliferation in lungs of ventilated and oxygen-treated preterm infants. Eur Respir J 23: 113-121, 2004.

46. Gao W, Mao Q, Feng A, Sun HM, Sun WK, Lu X, Su X and Shi Y: Inhibition of pre-B cell colony-enhancing factor attenuates inflammation and apoptosis induced by pandemic H1N1 2009 in lung endothelium. Respir Physiol Neurobiol 178: 235-241, 2011.

47. Ming GF, Ma XH, Xu DM, Liu ZY, Ai YH, Liu HX and Shi ZH: PBEF promotes the apoptosis of pulmonary microvascular endothelial cells and regulates the expression of inflammatory factors and AQP1 through the MAPK pathways. Int J Mol Med 36: 890-896, 2015 